\title{
EMBARAZO EN RECEPTORAS DE TRASPLANTE RENAL
}

Lupe Eugenia Diaz T, Deysy Aleida Mora S, Sandra Milena Rincón T, Sonia Liliana Rivera B*

\section{Resumen}

La investigación describe 21 embarazos en 19 pacientes receptoras de trasplante renal de la Clínica San Pedro Claver de Bogotá, durante el período comprendido entre 1992 y 2003. Se destaca la importancia del enfermero como parte del equipo multidisciplinario tratante de este grupo de pacientes, lo cual implica poseer los conocimientos que permitan sustentar las intervenciones tendientes a favorecer la calidad de vida. Se propone este tema como eje de la investigación, para contribuir al desarrollo de posteriores estudios que permitan el crecimiento del desempeño de la enfermería en esta área.

Se revisaron las historias clínicas anotando lo pertinente en la hoja de recolección de datos y además se obtuvo información directa de las pacientes. Se halló que en la etapa post-trasplante 11 pacientes presentaron episodios de rechazo previos al embarazo, la función menstrual se mantuvo aún en presencia de insuficiencia renal crónica en ocho, mientras que en cinco se restauró al mes y seis hasta un año después.

En lo referente al tiempo transcurrido entre trasplante y embarazo se observó un promedio de 3,35 años con intervalo de ocho meses a siete años. Los embarazos exitosos fueron en quienes tuvieron una creatinina menor a $1,5 \mathrm{mg} / \mathrm{dl}$. Se encontraron cinco pacientes con episodio de rechazo postparto; una presentó elevación de los niveles de creatinina a 6,6 mg/dl al momento del parto, requiriendo hemodiálisis dos semanas después de éste, otra aumentó la creatinina a 3,3 mg/dl al momento del parto con hemodiálisis durante un mes en el postparto, las otras tres necesitaron hemodiálisis después del primero, segundo y cuarto año respectivamente.

En cuanto a la terapia inmunosupresora no se evidenciaron cambios en la dosis e intervalo durante el embarazo. El factor comórbido más relevante fue la hipertensión arterial seguida de preeclampsia. Se presentaron 17 nacimientos, diez a término, siete pretérmino, un óbito y tres abortos. La vía del parto más frecuente fue la cesárea. Según lo observado en este grupo de estudio se puede decir que la gestación no parece causar deterioro del injerto cuando el embarazo se inicia con una función renal estable. Sin embargo, el embarazo en pacientes con trasplante renal debe ser considerado como de alto riesgo y controlado por un equipo multidisciplinario.

Palabreas clave: riñón, trasplantes y embarazo, embarazo, gestación, trasplante renal.

\section{Introducción}

El trasplante de órganos y tejidos constituye una disciplina nueva en el mundo. De hecho, la mayor parte de su desarrollo ha ocurrido en los últimos 30 años. Sin embargo, ya dejó de ser una actividad experimental para constituirse en parte básica del armamentario terapéutico disponible en la actualidad para el tratamiento del paciente con insuficiencia terminal de un órgano.

Enfermeras estudiantes III semestre Fundación Universitaria de Ciencias de la Salud, Hospital de San José. Facultad de Enfermería, Enfermeras especialistas en nefrología y urología 2004.

Este trabajo fue realizado con la Asesoría Temática de la Dra. Claudia Abreo, jefe de enfermeras de la sección de trasplante renal de la Clínica San Pedro Claver, en el período comprendido entre 1992 y 2003.
En el caso de enfermos con insuficiencia renal crónica (IRC), el trasplante ofrece significativas ventajas en lo que se refiere a sobrevida y, de modo particular, a calidad de vida de los pacientes, cuando se compara con otra terapia de reemplazo. Para muchos el trasplante significa la única posibilidad de supervivencia.

Después del trasplante renal la función menstrual retorna a la normalidad siendo posible la gestación. Una de cada 50 mujeres queda embarazada y en la mayoría de los casos el resultado es exitoso para la madre, el niño y el injerto. A pesar de ello, se debe llevar un seguimiento estricto de la función renal antes del embarazo, así como de los efectos de la terapia inmunosupresora. 
Datos estadísticos demuestran que nuestro país entró en el uso del trasplante renal como terapia de elección en IRC. Es así como en la actualidad el programa de trasplante renal de la Clínica San Pedro Claver de Bogotá, cuenta con 480 pacientes en seguimiento, de los cuales el $64 \%$ son hombres y $36 \%$ mujeres.

\section{Materiales y métodos}

La presente investigación describe 21 embarazos en 19 receptoras de trasplante renal de la Clínica San Pedro Claver durante el período comprendido entre 1992 y 2003. El estudio utiliza un diseño descriptivo retrospectivo. En cuanto a los criterios de inclusión, se seleccionaron historias clínicas que permitieron obtener la información contenida en la hoja de recolección de datos así como la aceptación expresa de las pacientes para participar en la investigación. Respecto a los criterios de exclusión fueron las historias clínicas ilegibles o con datos incompletos.

Se diseñó una hoja de recolección de datos que incluyó identificación, información acerca del trasplante, terapia anticonceptiva, función renal y tratamiento farmacológico antes, durante y después del embarazo, complicaciones comórbidas durante el embarazo, desenlace del mismo, terapia de reemplazo renal posterior al embarazo y la actual.

\section{Resultados}

La etiología de IRC con más prevalencia fue la hipertensión arterial (HA) (47\%), seguida de la no aclarada $(38 \%)$, en menor porcentaje la glomerulonefritis $(10 \%)$ y el reflujo ureterovesical (5\%). El origen del trasplante fue en su mayoría de donante cadavérico con un $71 \%$ y $29 \%$ de donante vivo. Teniendo en cuenta la información adecuada ante la situación médica, se evidenció que la habían recibido sobre planificación un $85 \%$, todas recibieron información acerca del trasplante y sobre el riesgo que trae el embarazo, el 90,5\%. Dentro de los antecedentes patológicos previos se vió que el 80,95\% corresponde a HA.
La composición por edad al momento del embarazo tuvo un promedio de 28,5 años con una desviación estándar de 2,5 años y un rango de 19 a 40 años. Con relación al número de embarazos post-trasplante renal se evidenció que el promedio de embarazos fue de 1,48 con una desviación estándar de 0,57 y un rango de uno a cuatro. Con referencia a la edad gestacional al momento del parto el promedio en semanas fue de 32.06 con una desviación estándar de 5,65 semanas y un rango de 8 a 39 .

Respecto a la frecuencia de episodios de rechazo el $52 \%$ de la muestra lo presentó. Se observó que la función menstrual no se alteró en un $38,10 \%$, en $28,58 \%$ se restauró al mes del trasplante, $14,28 \%$ al año, 9,52\% a los a 1 os tres meses, $4,76 \%$ a $\operatorname{los}$ dos y cinco meses. (Figura 1).

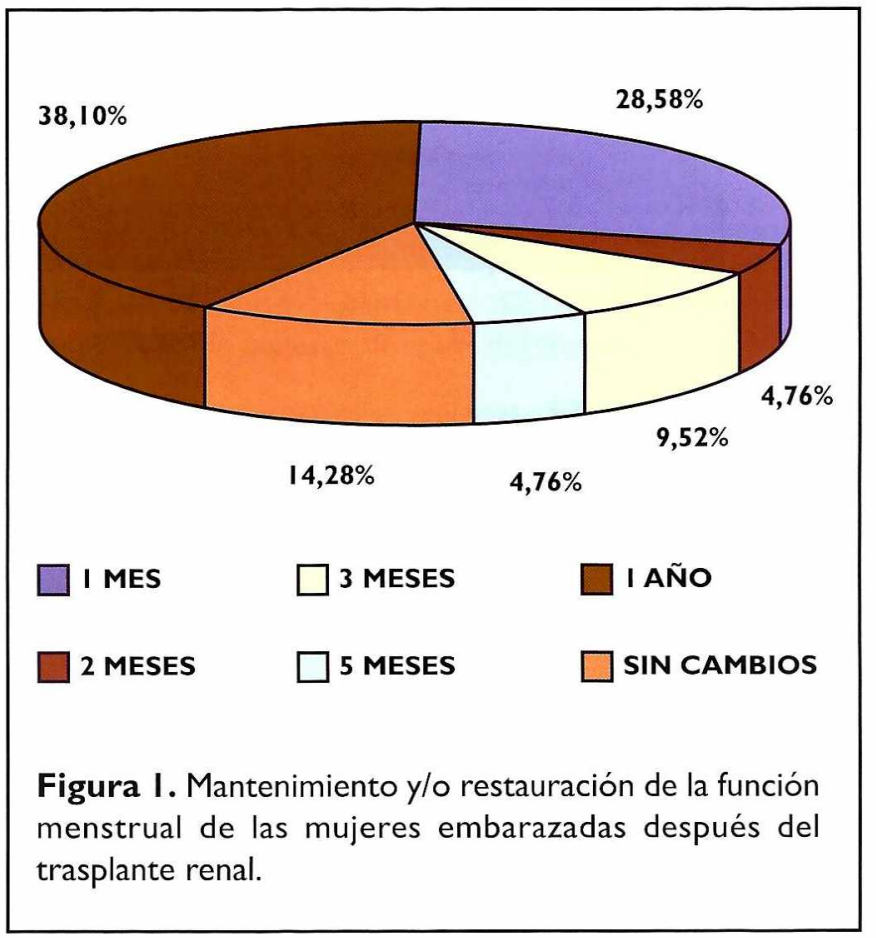

En cuanto a los métodos de planificación familiar se observó que 52,38\% los utilizó (Figura 2). E1 método más común fue el preservativo con un $38,09 \%$, seguido de los anovulatorios y los métodos de barrera en un $9,52 \%$ y por último los anovulatorios en un 4,76\%. (Figura 3 ). 

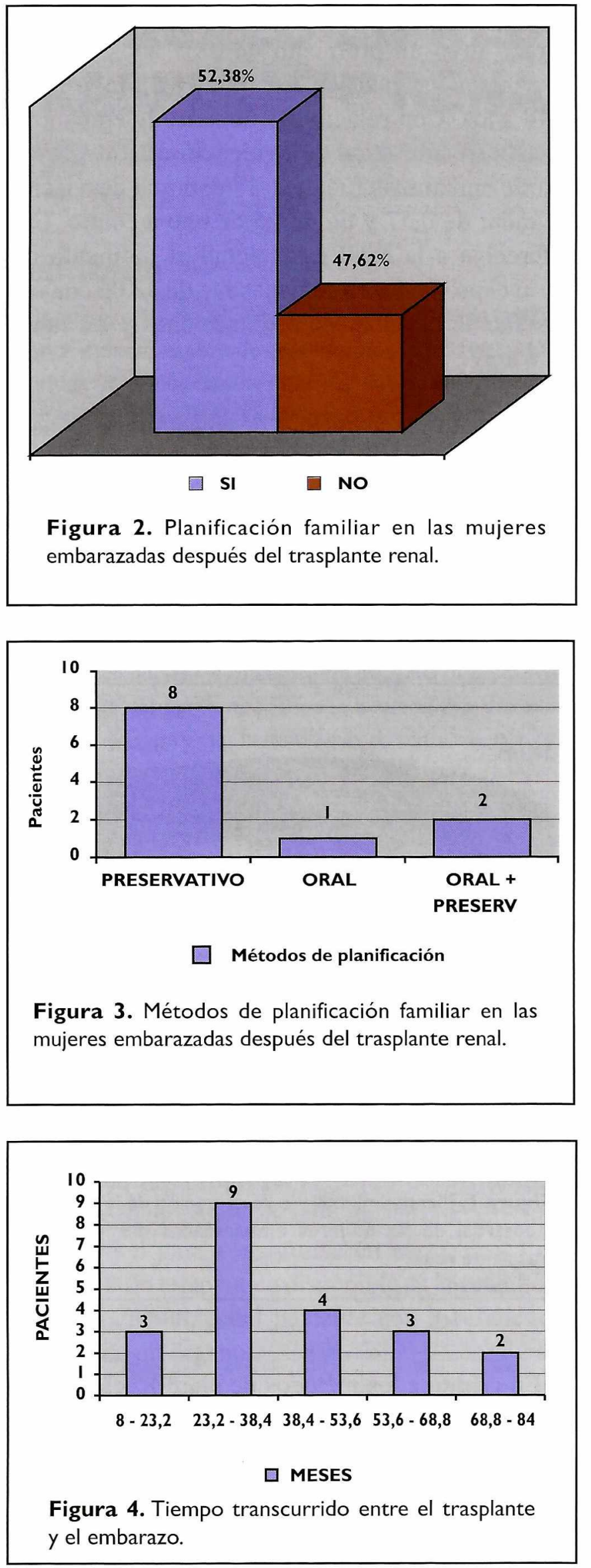

El tiempo transcurrido entre el trasplante renal y el embarazo tuvo un promedio de 40,2 meses con una desviación estándar de 8,36 y un rango de 8 a 84 meses (Figura 4).

La función renal con respecto a la creatinina, (Figura 5) tuvo un promedio antes del embarazo de $1,26 \mathrm{mg} / \mathrm{dl}$; al mes del embarazo $1,18 \mathrm{mg} / \mathrm{dl}$; al momento del parto $1,59 \mathrm{mg} / \mathrm{dl}$; un mes después del parto $1,35 \mathrm{mg} / \mathrm{dl}$; a los tres meses post-parto $1,29 \mathrm{mg} / \mathrm{dl}$ y al año $1,33 \mathrm{mg} / \mathrm{dl}$.

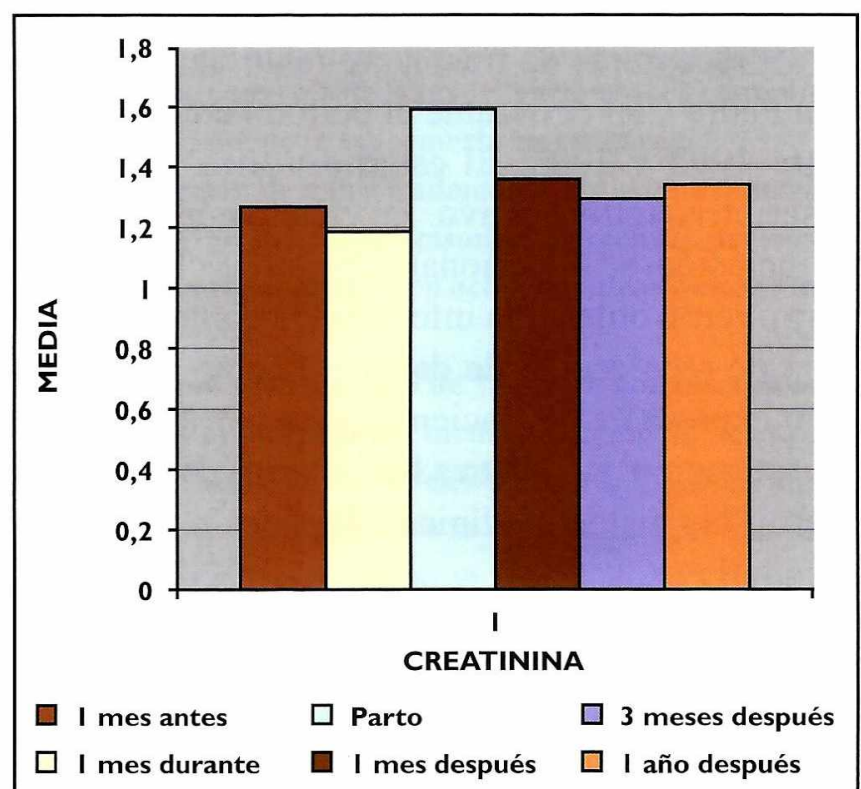

Figura 5. Media de la creatinina en las embarazadas después del trasplante renal.

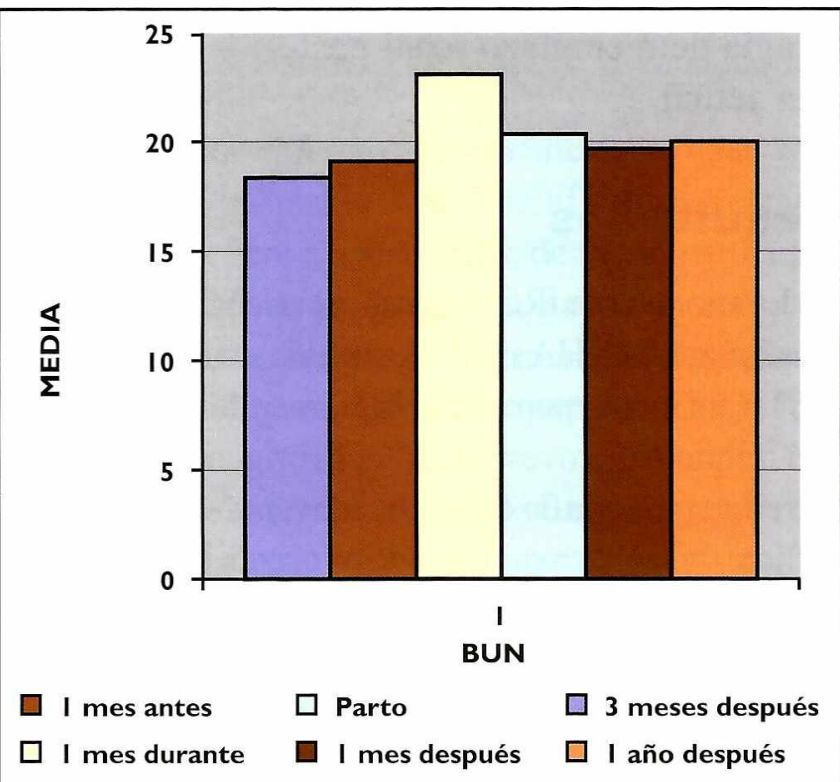

Figura 6. Media del BUN en las embarazadas después del trasplante renal. 
Respecto al BUN, el promedio antes del embarazo, fue de 18,34 mg/dl; al mes del embarazo 19,09 $\mathrm{mg} / \mathrm{dl}$; al momento del parto $23,08 \mathrm{mg} / \mathrm{dl}$; un mes después del parto $20,32 \mathrm{mg} / \mathrm{dl}$; a los tres meses postparto $19,66 \mathrm{mg} / \mathrm{dl}$ y al año $19,99 \mathrm{mg} / \mathrm{dl}$ (Figura 6).

Dentro de la función renal no se analizó la proteinuria por falta de datos en las historias clínicas que permitieran establecer una comparación entre los diferentes períodos observados.

Respecto al tipo de terapia inmunosupresora utilizada se encuentra que el 80,95\% emplearon triple (ciclosporina + prednisona + azatioprina), el 14,28\% utilizó doble (prednisona y ciclosporina) y el 4,77\% con prednisona y azatioprina. (Figura 7). En cuanto a las dosis de azatioprina antes, durante y después del embarazo, no se modificó en el 88,24\% y el 11,76\% cambió antes y durante el embarazo. Con respecto a las dosis de ciclosporina se observó que el 90\% mantuvo una dosis constante antes, durante y después del embarazo y el 10\% la disminuyó durante el embarazo.

Las dosis de prednisona administradas no presentaron cambios en el 80,95\%, mientras que al 14,25\% la disminuyeron durante durante el embarazo y al $4,8 \%$ la aumentaron después del embarazo.

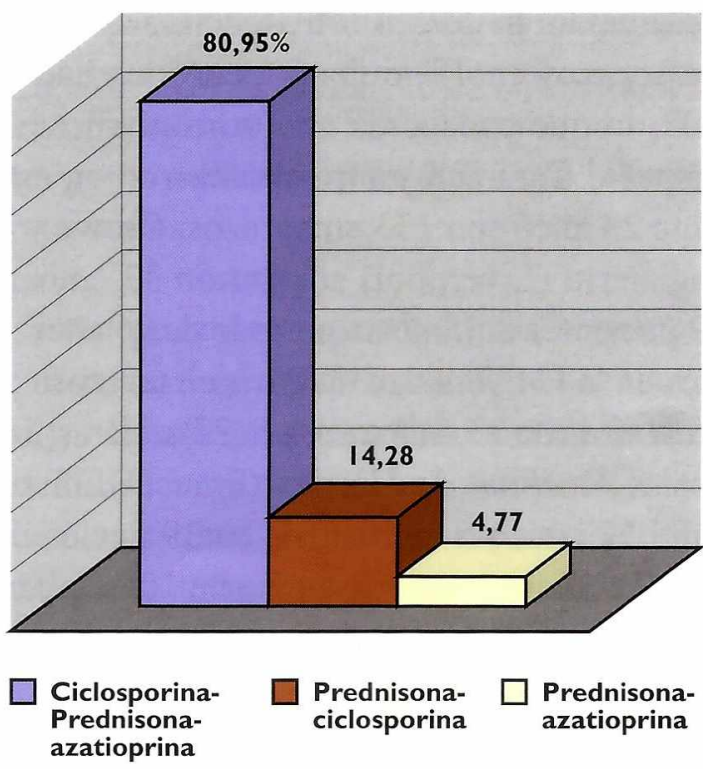

Figura 7. Terapia inmunosupresora en las mujeres embarazadas después del trasplante renal.
Las complicaciones comórbidas más frecuentes durante la gestación fueron la HA en el $42,85 \%$, durante el primer trimestre y $61,90 \%$ en el segundo y tercero la preeclampsia no se observó durante el primer trimestre, en el segundo y tercero se encontró en un 23,80\%; no se observó eclampsia, síndrome Hellp ni diabetes gestacional en ningún trimestre. Respecto a la infección urinaria se detectó en el 38,09\% en el primer trimestre y $9,52 \%$ en el segundo y tercero; las Infecciones vaginales ocuparon un 19,04\% durante el primer trimestre, no se presentaron en el segundo y $4,76 \%$ en el tercero. (Figura 8 ).

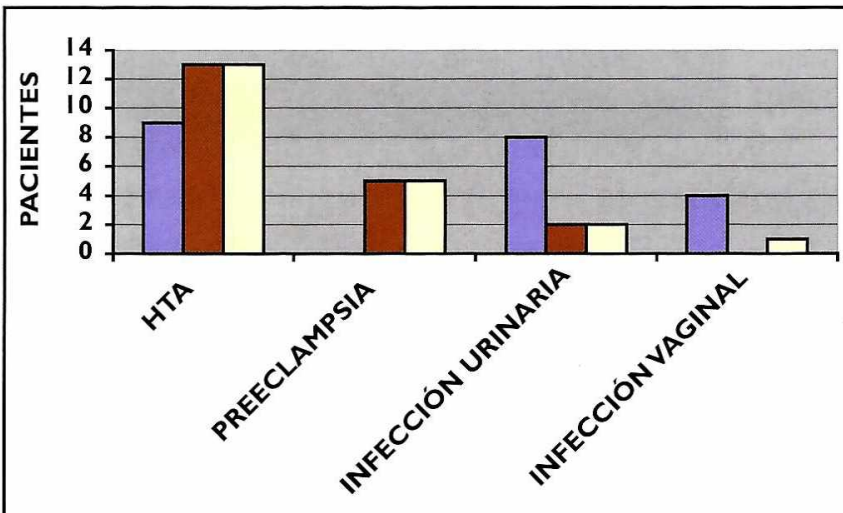

口I TRIMESTRE $\square 2$ TRIMESTRE $\square 3$ TRIMESTRE

Figura 8. Complicaciones mórbidas durante el embarazo en mujeres después del trasplante renal.

El desenlace del embarazo fue $48 \%$ a término, el $33 \%$ pretérmino, 14\% aborto y un 5\% óbito (Figura 9).

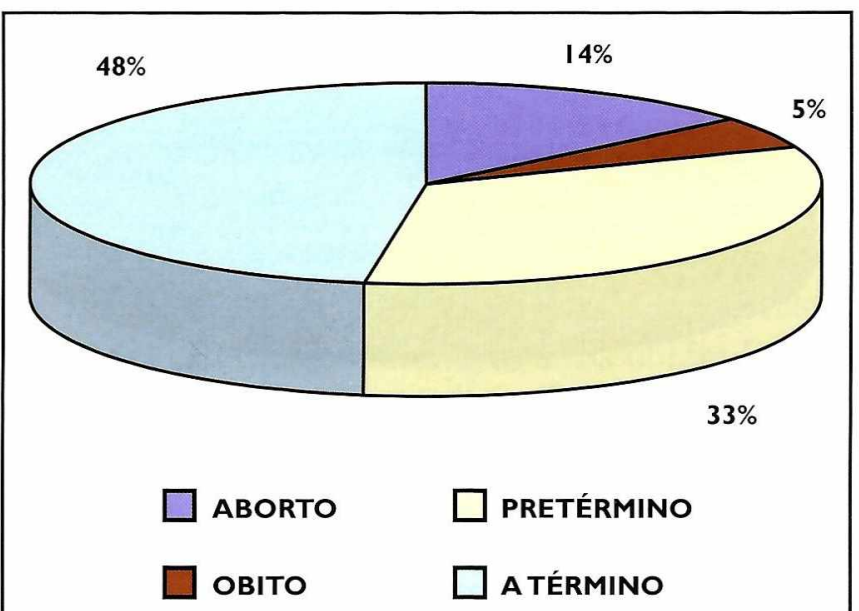

Figura 9. Desenlace del embarazo en mujeres después del trasplante renal. 
La vía del parto más utilizada fue la cesárea en el 94,44\% (17) y en un menor porcentaje la vaginal $5,56 \%$ (1). (Figura 10).

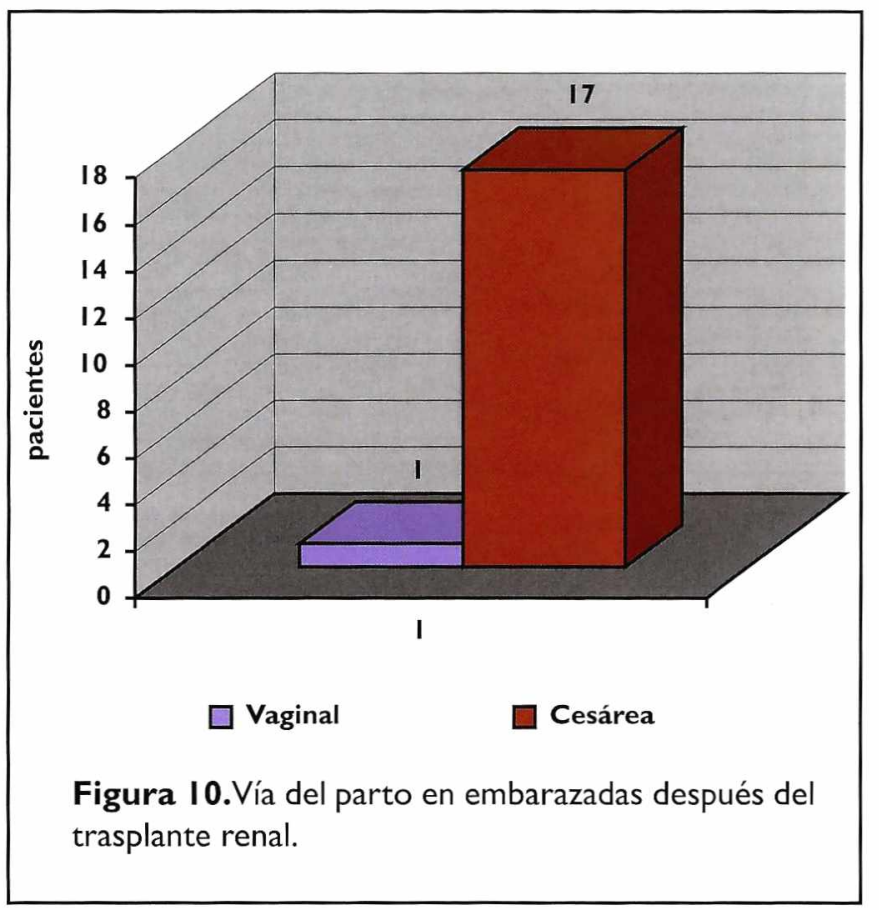

E1 24\% presentó episodios de rechazo y el $76 \%$ no (Figura 11). Así mismo, de los 21 embarazos, 16 no requirieron terapia dialítica postparto mientras cinco sí, siendo la más utilizada la hemodiálisis. (Figura 12).

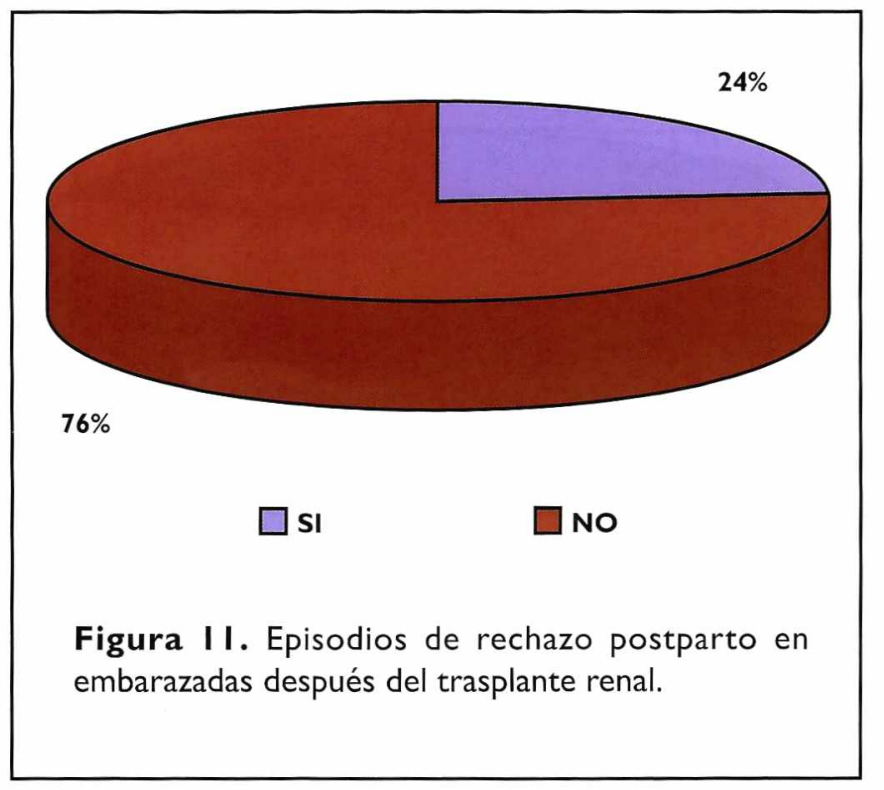

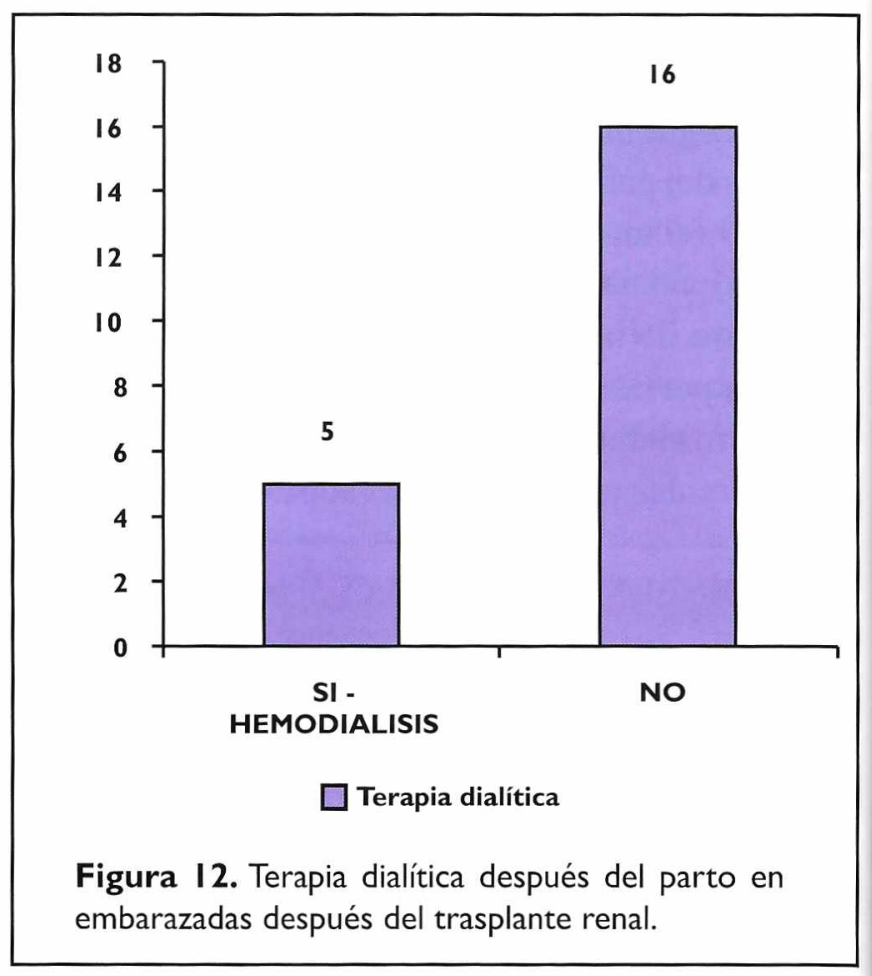

\section{Discusión}

El trasplante renal mejora la calidad de vida de las mujeres en edad fértil, ya que permite una recuperación del estado endocrino y la función renal. Mundialmente se han reportado alrededor de 2.000 embarazos en receptoras de trasplante renal.

Se analizó en forma retrospectiva el curso de 21 embarazos en 19 mujeres ocurridos entre 992 y 2003, lo que concuerda con otras publicaciones. En España, Cararach y otros realizaron un estudio durante 23 años con 133 embarazos; Crowe y otros en Inglaterra (Liverpool) analizaron 33 embarazos en 29 pacientes durante un período de 26 años; Rieu y otros en la Universidad de Paris en un lapso de 25 años observaron 33 embarazos en 22 mujeres; Touraine en el Hospital de Herriot (Francia), investigó durante 17 años 45 embarazos en 39 pacientes; en Alemania, Kainz y otros estudiaron 100 embarazos en 84 pacientes en un periodo de 6 años.

Los extremos y el promedio de edad de este estudio son similares a los reportados por Crowe y otros quienes encontraron edades comprendidas entre 19 y 39 años, con un promedio de 29 años. 
En cuanto al rechazo previo al embarazo, el estudio muestra que 11 (52\%) lo presentaron y 10 $(48 \%)$ de ellas no, según la guía EBPG (European Best Practice Guidelines for Renal Trasplantation). La incidencia de rechazo agudo no es mayor que en trasplantadas no embarazadas. La incidencia de rechazo agudo durante el embarazo y tres meses después del parto varía entre 9 y $14,5 \%$ en las series publicadas.

En cuanto al mantenimiento de la función menstrual, en el estudio se encuentra que el $38,09 \%$ la mantuvo, aún en IRC; mientras que el 28,57\% la restauró al mes y el 33,33\% restante después del mes postrasplante.

Al existir la falla renal se presentan alteraciones a nivel reproductivo manifestadas por amenorrea, anovulación, cambios en los niveles de FSH, LH, progesterona, estradiol y prolactina. Casi el 50\% de mujeres con IRC tienen hiperprolactinemia y en consecuencia el embarazo es poco frecuente y cursan con niveles elevados de creatinina y BUN.

Posterior al trasplante renal eficaz se restaura la fertilidad dentro de un promedio de seis meses y cerca de una de cada 50 mujeres queda embarazada. Los resultados en cuanto a la terapia anticonceptiva muestran que el 53,38\% utiliza algún método, siendo el de barrera $(38,82 \%)$ el más usado, seguido del $9,5 \%$ anovulatorios y un $9,5 \%$ la combinación entre barrera y anovulatorios y un 4,8\% usó los anticonceptivos orales.

Existen pocas recomendaciones respecto a la anticoncepción en trasplantadas, sin embargo se debe aconsejar a todas aquellas en edad fértil sobre las posibilidades y riesgos de un embarazo después del trasplante renal. La anticoncepción se debe comenzar inmediatamente después del trasplante ya que los ciclos ovulatorios pueden reiniciarse en los primeros dos meses.

El reducido uso del dispositivo intrauterino se asocia con un elevado riesgo de infección y su eficacia se ve disminuida por los efectos antiinflamatorios de los inmunosupresores. Además pueden agravar los problemas menstruales y ocultar signos y síntomas de una gestación.

En cuanto al tiempo transcurrido entre el trasplante y el embarazo se observó que el promedio fue de 40,2 meses con un intervalo entre ocho meses y siete años. Esto se relaciona con el estudio de Saito y otros en Japón donde encontraron entre dos y cinco años postrasplante; Rieu en Francia encontró entre 1,5 y 2,5 años; Touraine en Francia entre 2 y 10 años y Crowe entre 5 y 10 años.

La EBPG sugiere esperar dos años después del trasplante para concebir, ya que se encontró que el lapso transcurrido entre el trasplante y el embarazo era un predictor significativo para el resultado esperado. La razón para esta recomendación es que si la función del injerto se mantiene durante 24 meses, existe una alta probabilidad de que se mantenga durante los cinco años siguientes.

Se encontró que la mayoría de los embarazos con finales exitosos fueron en quienes mantuvieron buena función renal (creatinina sérica $<1,5 \mathrm{mg} / \mathrm{dl}$ ). De las cinco que presentaron rechazo posparto, cuatro progresaron a daño renal requiriendo terapia dialítica después del embarazo y una posterior al aborto. De éstas, una elevó la creatinina $(6,6 \mathrm{mg} / \mathrm{dl})$ al momento del parto requiriendo hemodiálisis en el postparto durante dos semanas, otra también la elevó $(3,3 \mathrm{mg} / \mathrm{dl})$ en el parto necesitando hemodiálisis durante un mes. Una presentó elevación de la creatinina sérica $(2,1 \mathrm{mg} / \mathrm{dl})$ al año del parto, requiriendo terapia dialítica 4 años después de éste y dos no mostraron alteración de creatinina durante el embarazo, pero requirieron terapia dialítica dos y cuatro años postparto respectivamente. Los resultados del estudio son similares a los obtenidos por Crowe, quien analizó los valores en preconcepción, parto, y tres a seis meses postparto, encontrando embarazos exitosos con niveles de creatinina menores de $1,7 \mathrm{mg} / \mathrm{dl}$, en contraste con aquellas con creatinina superior a $2,2 \mathrm{mg} / \mathrm{dl}$, quienes progresaron a daño renal requiriendo terapia de reemplazo a los dos años. 
En el estudio se observó que el comportamiento del BUN fue similar al de creatinina pero no se encontraron publicaciones que permitieran establecer una comparación entre estos parámetros. Otros estudios analizan la función renal con datos comparativos de creatinina sin especificar el lapso en que fueron observados. Entre estos están: Rieu quien encontró que la función renal permanecía estable durante el embarazo en todas las mujeres, incluyendo las que tenían creatinina anormal antes de la concepción.

Touraine encontró 29 pacientes con creatinina menor de $1,2 \mathrm{mg} / \mathrm{dl}, 13$ con una elevación moderada $(1,2-2,2 \mathrm{mg} / \mathrm{dl})$ y tres mantuvieron niveles entre 2,2 y $3,3 \mathrm{mg} / \mathrm{dl}$. En el estudio realizado por Saito se concluyó que la creatinina sérica era un indicador de la función renal y no se deterioró durante el embarazo en receptoras de trasplante renal, pero no presentó valores de referencia.

La terapia inmunosupresora más usada fue ciclosporina, azatioprina y prednisona en 17 pacientes $(80,95 \%)$, mientras la conjugación ciclosporina y prednisona solo se utilizó en tres pacientes $(14,28 \%)$ y prednisona y azatioprina en una paciente $(4,76 \%)$. Respecto a la dosificación, el 14,28\% requirió aumento de la misma, mientras que el $37,82 \%$ permaneció con dosis de mantenimiento. No fue necesario alterar la inmunosupresión en la concepción o después de ella, ya que la función renal permaneció estable. Danovich planteó que se deben aumentar las dosis de esteroides para cubrir el estrés del parto y prevenir el rechazo postparto. Comparado con nuestra investigación, solo se incrementó la dosis de esteroides al momento del rechazo postparto en uno de los casos.

Armenti refiere que existe poca información acerca de la nuevas combinaciones de medicamentos, por lo tanto, se deben realizar estudios que presenten información y lineamientos con respecto a estos regímenes. La inmunosupresión debe estar a niveles de mantenimiento al inicio del embarazo y durante su transcurso.
En el estudio se encontraron como factores comórbidos predominantes en el primer trimestre a la HA con un total de nueve pacientes $(42,85 \%)$ tratadas con captopril, alfa-metildopa, propanolol, verapamilo; en segundo lugar las infecciones de las vías urinarias en ocho pacientes $(38,09 \%)$, tratadas con ampicilina y ampicilina - sulbactam y por último las infecciones vaginales en menor proporción en cuatro pacientes $(19,04 \%)$, manejadas con metronidazol. Durante el segundo y tercer trimestre prevalecen en su orden de ocurrencia: $\mathrm{HA}$, preeclampsia, infecciones de vías urinarias y por último las infecciones vaginales.

La EBPG expone que aproximadamente el 50\% de las pacientes con ciclosporina presentan HA antes del embarazo, y este valor aumenta durante el embarazo entre 62 y 70\%, con incidencia de preeclampsia del $27 \%$ con ciclosporina y $23 \%$ con ciclosporina emulsión. La ciclosporina aumenta la producción de tromboxano y endotelina, implicados en la patogénesis de la HA. Las embarazadas con trasplante renal tienen alto riesgo de infección, especialmente bacteriana. De hecho el $40 \%$ la tienen en el tracto urinario.

Davison recomienda cultivos mensuales de orina. En caso de bacteremia asintomática la paciente puede tratarse por dos semanas profilácticamente hasta el final del embarazo. Crowe presentó la descripción de los factores comórbidos más frecuentes, encontrando en primera instancia HA seguida de preeclampsia y por último las infecciones vaginales.

En la presente investigación hubo 17 nacimientos sanos. De estos, $10(47,2 \%)$ fueron partos a término, $7(33,3 \%)$ pretérmino ( $<37$ semanas), con un promedio de edad gestacional al momento del parto de 35,2 semanas y un rango de 28 a 39 semanas. Hubo tres abortos espontáneos $(14,29 \%)$, un óbito $(4,76 \%)$, no hubo abortos terapéuticos ni embarazos ectópicos y la incidencia de abortos naturales fue baja.

En el estudio de Rieu se encontró una alta incidencia de pretérmino con un promedio de edad gestacional al momento del parto de 36,1 semanas con un rango entre 31 y 40 semanas. Hubo un óbito fetal 
seguido de un aborto. Por otra parte Crowe señala también una alta incidencia de partos pretérminos con una edad gestacional de 34,2 semanas, cuatro abortos naturales, un óbito y un neonato que murió sin causa conocida. Touraine muestra que hubo nueve nacimientos entre las 32 y 33 semanas, nueve entre 34 y 35 y 27 a las 36.

Davison informa una serie importante de embarazos después del trasplante renal: 2.382 en 2.409 mujeres con $20 \%$ de abortos espontáneos, comparables con la población general. Las gestaciones que progresaron más allá del primer trimestre terminaron con éxito. Sin embargo, la incidencia de partos pretérmino fue del $20 \%$. La literatura muestra problemas como prematurez (45-60\%) y bajo peso para la edad gestacional (20-30\%), atribuible al efecto farmacológico de inmunosupresores y la relación de estos con la función renal y/o con la presencia de HA.

En esta investigación se encontró que la vía del parto más frecuente fue la cesárea en 17 pacientes $(94,44 \%)$ y vaginal una $(5,56 \%)$, lo que concuerda con otros estudios como el realizado por Crowe quien encontró que el trasplante renal no constituye un obstáculo para el parto vaginal. Sin embargo, las tasas de cesárea en estas circunstancias pueden ser altas $(25$ - 85\%). Según Rieu el 78\% fueron cesáreas y el $22 \%$ partos vaginales. Touraine enuncia que el parto vaginal es factible en pacientes postrasplante renal, mostrando que la cesárea es usada entre 25 y $80 \%$ en caso de HA severa, deterioro de la función renal o retardo de crecimiento intrauterino.

Por último, de la muestra estudiada $16(76 \%)$ no requirieron terapia dialítica, mientras que cinco $(24 \%)$ regresaron a hemodiálisis, tres de ellas $(14,4 \%)$ perdieron el injerto y continuaron en hemodiálisis, mientras que dos $(9,6 \%)$ fueron retrasplantadas. Danovich recomienda que durante los tres primeros meses después del parto las trasplantadas deben continuar en estrecha vigilancia en la unidad, ya que la función renal puede verse deteriorada y en o ocasiones llevar a síndrome hemolítico urémico. En el estudio realizado por Rieu se concluyó que no hubo episodios de rechazo agudo y solo una mujer experimentó nefropatía de trasplante y volvió a hemodiálisis cinco años después del parto.

\section{Conclusiones}

Esta investigación describe embarazos exitosos en mujeres receptoras de trasplante renal, concluyendo que la gestación no parece causar deterioro de la función del injerto cuando el embarazo se inicia con una función estable del mismo. Sin embargo, en este estudio la probabilidad de haber fallado en detectar diferencias antes y después del embarazo puede deberse a dificultades diagnósticas o a la ausencia de verdaderas diferencias.

El comportamiento de la función renal (creatinina) no cambió en la muestra estudiada, excepto en cuatro que presentaron rechazo postparto con aumento en los valores de creatinina $>2 \mathrm{mg} / \mathrm{dl}$, requiriendo terapia dialítica.

En algunas pacientes la función menstrual no se alteró aún con IRC, mientras que en el resto de la muestra dicha función se recuperó después del trasplante.

El embarazo debe considerarse de alto riesgo y controlarse por un equipo multidisciplinario, que vigile estrechamente la función renal, presión arterial, condiciones fetales, y en especial la detección temprana de signos de rechazo.

La terapia anticonceptiva no se utilizó durante el tiempo recomendado para asegurar la supervivencia del injerto. A pesar de que las pacientes habían recibido información oportuna, quedaron en gestación antes del plazo determinado.

El análisis de la función renal con respecto al BUN no es relevante porque este se ve influenciado por la masa muscular, actividad física e ingesta proteica, por lo cual se debe tener en cuenta es la creatinina y su depuración. En la muestra estudiada la terapia inmunosupresora no tuvo cambios en cuanto a dosis e intervalo durante el embarazo. 
Para aumentar la probabilidad de conseguir un embarazo a término se deben tener en cuenta los factores de pronóstico favorables, tales como: evolución satisfactoria en los dos últimos años post-trasplante, ausencia de proteinuria o hipertensión, datos de rechazo, dilatación pielocalicial, creatinina menor o igual a $1,5 \mathrm{mg} / \mathrm{dl}, \mathrm{BUN}<22 \mathrm{mg} / \mathrm{dl}$ e inmunosupresores en dosis de mantenimiento.

En la muestra estudiada, durante el embarazo se exacerbaron ciertos factores comórbidos, siendo la más relevante la $\mathrm{HA}$, que conlleva al riesgo de rechazo crónico.

Aunque no existe una evidencia teórica que contraindique el parto vaginal, la cesárea fue la vía más utilizada en estas pacientes. En nuestra muestra se encontró un porcentaje similar de recién nacidos a término y pretérmino, sin ninguna relación importante entre la inmunosupresión y la HA sobre el desarrollo del feto.

\section{Lecturas recomendadas}

Armenti V. Embarazo y trasplante. En: trasplante de órganos. México: JGH 1999.

Cararach V et al. Pregnancy after renal transplantation 25 years experience in Spain. Department of obstetrics and gynecology. Hospital clìnic and provincial, Faculty of Medicine, University of Barcelona, Spain. Br J Obstet Gynecol 1993; feb.

Crowe A V et al. Pregnancy does not adversely affect renal transplant function, from the renal unit and renal transplant unit, Royal. Liverpool University Hospital, Association of Physicians, 1999.

Danovitch, Gabriel. Trasplante Renal. 3 ed. Madrid; Marbán, 2002.

Davison JM. Pregnancy in renal allograft recipients: problems, prognosis, practicalities. Clin Obst Gynecol 1994; 8: 501-525.

Expert Group on Renal Transplantation-EBPG. European best practice guidelenes for renal transplantation. Section IV: long-term management of the transplant recipient. Pregnancy in renal transplant Asociation-European Renal Association.

Hou S. Pregnancy in chronical renal insufficienty and endstage renal diseas Am J Kidney 1999; 33: 235 - 252.

Kainz A et al. Review of the course and outcome of 100 pregnancies en 84 women treated with tacrolimus. En: Transplantation, Center Fujisawa, Germany, Dic 27:70. 2000. pàg. 1718-21.

Murray JE et al. Successful pregnancies after renal trasplantation. N Engl J Med 1993; 269: 341-343

Rieu P et al. Thirty-three pregnancies in a population of 1725 Renal transplant patients. En: transplantation proceedings: Elservier Science 1997. 29,2459-60.

Saito S Et al. Successful pregnancy in renal trasplante recipients. Acta Med Okayama 1993.

Touraine JL Pregnancy in Renal Transplant patients: 45 case Reports. En: transplantation proceedings: Elservier Science 1997. 29,2459-2460.

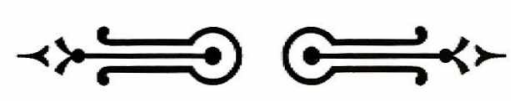

